\title{
Behavioral Stock Portfolio Optimization through Short-Selling
}

\author{
Kuo-Hwa Chang, Michael N. Young, Chien-Chih Liu, and Hao-Ping Chung
}

\begin{abstract}
With the introduction of behavioral stocks (B-stocks), investors now have an alternative profitable investment option by exploiting the positive effect (positive cumulative abnormal return CAR) of irrational behaviors to stock price movements. Currently, all work on B-stocks only consider the cause-and-effect relations that expect B-stocks to have positive CARs after some time following the spotting of their respective causes. Thus, this study finds the other side and exploits the cause-and-effect relations that expect B-stocks to have negative CARs after some time following the spotting of their respective causes. We call this short-sell B-stocks. Accordingly, this study proposes a scenario-based mixed integer program that considers the short-selling of B-stocks under the inverse version of the safety-first portfolio selection model to maximize the negative price change of $B$-stocks within the portfolio. The result shows that the short-sell portfolio can outperform the market portfolio significantly; this also indicates that the proposed investment strategy can be another alternative profitable investment option that investors can exploit.
\end{abstract}

Index Terms-Behavioral stocks, short-sell B-stocks, holding periods, investments, portfolio selection, short-selling

\section{INTRODUCTION}

With the popularity of behavioral finance, recent works by [1]-[4] introduced behavioral stocks (B-stocks). B-stocks are stocks which are affected by the collective irrational behaviors of investors. They study the connection between the irrational behaviors and the stock price movements. By identifying the cause and effect relationship between the bias and the stock returns, they determined which stocks are significantly affected by irrational behaviors. In other words, by identifying the respective operational definitions (ODs) of irrational behaviors, the cause-and-effect relationships between the irrational behavior and stock returns can be determined. These ODs reflect when a bias occurs, what is the bias trigger is (cause) and what happens (effect) after this

Manuscript received March 1, 2018; revised April 28, 2018. This work was partially supported by the Ministry of Science and Technology, Taiwan, ROC, under the grant contract MOST 103-2221-E-033 -053.

Kuo-Hwa Chang is with Chung Yuan Christian University, Departmen of Industrial and Systems Engineering, 200 Chung Pei Road, Chung Li District, Taoyuan City, Taiwan 32023 (e-mail: kuohwa@cycu.edu.tw).

Michael Nayat Young is with Chung Yuan Christian University, Department of Industrial and Systems Engineering, 200 Chung Pei Road, Chung Li District, Taoyuan City, Taiwan 32023. He is also with Mapúa University, 658 Muralla St., Intramuros, Manila 1002, Philippines (e-mail: michaelnayatyoung@gmail.com).

Chien-Chih Liu is with Chung Yuan Christian University, Department of Industrial and Systems Engineering, 200 Chung Pei Road, Chung Li District, Taoyuan City, Taiwan 32023 (e-mail: angelapollyontw@gmail.com).

Hao-Ping Chung is with Chung Yuan Christian University, Department of Industrial and Systems Engineering, 200 Chung Pei Road, Chung Li District, Taoyuan City, Taiwan 32023 (e-mail: ssica0611@yahoo.com.tw). trigger occurs. E.g., OD for over-reaction bias is defined as "a large positive (negative) price movement followed by a high negative (positive) cumulative abnormal return or CAR." from the descriptions of [5]-[6], [1]-[4]. From the definition of [7], [2]-[4] concluded that the OD for disposition effect bias is that investors tend to sell winners too soon and hold losers too long; considering the works of [8] and [9] on the synchronization between the price movement and volume of stocks to determine the disposition effect (serving as the cause) and the observation that when disposition effect winners (losers) are identified, their stock prices tend to depress (inflate) temporarily (serving as the effect) before reverting to its original trend. Thus, the definition of OD for disposition effect bias is "disposition effect losers (winners) followed by a large positive (negative) CAR." Further identifying other irrational behaviors or biases (e.g. representative bias [10], over-confidence [11]-[12], etc.) is possible in the future. By having these ODs, B-stocks can be identified; the corresponding information on their causes effect - likelihood-to-effect $\left(P^{B}\right)$ - time-to-effect ( $T$-days) patterns they possessed can be exploited to have superior portfolios. Subsequently, using these cause-effect- $T-P^{B}$ patterns [1]-[4] presented two portfolio selection procedures to exploit the resulting positive effect (positive $C A R$ ). [1]-[2] took advantage of the information; knowing when the effects will happen, they bought B-stocks a day before the effects occur then sold them when the effects occurred on the following day. On the other hand, [3]-[4] exploited the information on knowing when causes are spotted, the desired effects will occur $T$ days afterwards, with the corresponding likelihood-of-effect $P^{B}$ s, by buying B-stocks when their causes are spotted and then holding them until the corresponding $T$ days are reached. When the corresponding $T$ days are reached, B-stocks are sold to realize the profit. [1]-[4] showed that with the extra information on B-stocks, they were able to produce portfolios that possibly outperform traditional benchmarks specifically the mean-variance (MV) portfolios, generic safety-first (SF) portfolios, and market portfolios. Opportunely, their work only exploited the positive effect (positive CAR) information on B-stocks.

This study follows the respective ODs for over-reaction and disposition effect B-stocks and proposes a portfolio selection procedure that will exploit the adverse effect (negative CAR ) information on B-stocks; or short-sell B-stocks as we call it. Short-sell B stocks will provide investors with an alternative investment option that can probably outperform traditional benchmarks aside from the investment options provided by [1]-[4]. First, B-stocks having the desired cause-effect- $T-P^{B}$ patterns where the desired effect is to have a high negative $C A R$ after spotting its corresponding causes are identified. Second, by modifying 
the generic safety-first portfolio selection model to fit a short-selling strategy, a new portfolio selection procedure is modeled to exploit these short-sell B-stocks that will have negative CAR. Similar to [3]-[4], this study will consider the time epoch when the causes of short-sell B-stocks are spotted. From the possible short-sell B-stocks on a given day, the portfolio is rebalanced by shorting short-sell B-stocks and then closing them immediately after reaching their respective time-to-effects ( $T$-days). Lastly, to determine the potential profitability of the resulting short-selling portfolio, it will be compared to the market portfolio (benchmark).

This paper is organized as follows. In section 2, discussion on the determination of the short-sell B-stocks and the investment pool for each trading day. In section 3 , detailed information on data collection and application, presentation and analysis of empirical results. Then, in section 4, the conclusion, contribution, and possible future work are listed accordingly.

\section{Methodology}

In this study, we consider a daily investment in stock portfolios through short-selling. First, we identify the B-stocks that will have the negative effects of the collective disposition effect and over-reaction behavior of investors; or short-sell B-stocks intended for short-selling. These identified short-sell B-stocks are then included into the big pool. From this big pool, at each day of rebalancing the short-sell portfolio, we determine which B-stocks should be included in the investment pool (small pool). From this small pool, a proposed behavioral stock portfolio optimization model is used to have the optimal short-sell portfolio. Discussion of the corresponding procedures is in the succeeding subsections.

\section{A. Operational Definition (OD)}

Following the works [1]-[4], this study also focusses on the over-reaction and disposition effect B-stocks. B-stocks are determined based on the cause-and-effect relation and the corresponding time-to-effect ( $T$-days or time needed for the effect to occur) and likelihood-of-effect $\left(P^{B}\right.$ or probability that the effect will occur) between the collective irrational behavior of investors and the stock price movement. These cause-effect- $T-P^{B}$ patterns are determined based on the operational definitions (ODs) of each type of B-stock. As previously established, the definition of OD for over-reaction is "a large positive (negative) price movement followed by a high negative (positive) cumulative abnormal return (CAR)", and the definition of OD for the disposition effect is "disposition effect winners (losers) followed by high negative (positive) CAR". As mentioned, this study focuses on those B-stocks that will have the resulting negative effects, short-sell B-stocks. Therefore, the focus is on the causes that will lead to negative effects. The respective causes for over-reaction and disposition effect B-stocks are high positive price movement (a significant positive return rate) and disposition effect winner (from [2]-[4], disposition effect winners are stocks with a high positive cumulative return rate and highly abnormal trading volume). Spotting the over-reaction cause for short-sell B-stocks is straightforward, if there is a sudden increase in price in a given trading day (say, $+5 \%$ ) it is considered a possible cause, then if it is truly an over-reaction short-sell B-stock it will have a negative $C A R(C A R \leq-1 \%)$ after $T$ days. The identification of the disposition effect cause is more rigorous where we need to find a stock with a high cumulative return rate (say, 10\%) and highly abnormal trading volume, then if it is truly a disposition effect short-sell B-stock it will have a negative CAR $(C A R \leq-1 \%)$ after $T$ days. To spot the disposition effect short-sell B-stocks, this study follows [8] in identifying the existence of a disposition effect where a stock should have a high cumulative return (also a straightforward identification) and a trading volume which is considered abnormally high with respect to the market trading volume. The cumulative return is calculated as $R_{i}=\prod_{t=1}^{X}\left(R_{i t}+1\right)-$ 1 , where $R_{i t}$ is the return of stock $i$ at time $t$. Then an abnormally high trading volume is only considered when the average volume over $X$ period (say 30 days), $\overline{A V}$, calculated from $A V_{i t}=\widehat{V_{l t}}-\left(a+b V_{m, t}\right)$ is significant. $\widehat{V_{l t}}\left(V_{m, t}\right)$ is the observed volume of stock $i$ (market) at time $t$ which are derived from the relationship of the relative raw volume of stock and the relative raw volume of the market by the regression model, $V=a+b V_{m}+\sigma$. The relative raw volumes for each stock $i$ and the market are calculated as $V_{i t}=\frac{\text { volume at time } t}{\begin{array}{c}\text { moving average of the volume up to time } t \\ \text { market volume at time } t\end{array}}$ and $\quad V_{m, t}=$ $\overline{\text { moving average of the market volume up to time } t}$. As for the desired effect, say $C A R$ of at least at least $-1 \%$, the calculations is as follows. Considering the daily abnormal returns, $A R, C A R$ will be the summation of $A R$ over specific $T$ days or $C A R=\sum_{i=1}^{t} A R_{t} . A R_{t}$ is the abnormal return at time $t$ and $A R_{t}$ is calculated as $A R_{t}=R_{i t}-\beta_{i t} R_{M t}$ where $R_{i t}\left(R_{M t}\right)$ is the return rate of stock $i$ (market) at time $t$, and $\beta_{i t}$ is the corresponding beta.

To earn a profit in a short-selling strategy, only those over-reaction and disposition effect affected stocks that experience the effect of negative $C A R(C A R \leq-1 \%)$ are to be considered. Referring to the historical occurrences of the cause-effect relations for specific time-to-effect ( $T$ days) for each B-stock, if a stock's cause-effect pattern is significant, then this stock is considered to be a short-sell B-stock. To determine if a pattern is significant, the likelihood-to-effect of each stock $i\left(P_{i}^{B}\right)$ to hold such pattern should be higher than a threshold probability $(p)$. Consequently, a statistical test specifically the one-proportion test is performed with the null and alternative hypothesis $\left(H_{0}: P_{i}^{B} \leq p\right.$ and $\left.H_{a}: P_{i}^{B}>p\right)$ for each stock. For simplifying the rigorous tests, all stocks are tested using the time-to-effect ( $T$ days) from 1 to 20, but only the smallest or shortest $T$ days is considered.

\section{B. Investment Pool (Big Pool and Small Pool)}

From all the listed stocks on the Taiwan Stock Exchange (TWSE), the cause-effect- $T-P^{B}$ patterns between the irrational behaviors and the stock prices are analyzed to identify the short-sell B-stocks. These short-sell B-stocks then serve as the initial investment pool (big pool). Since this study considers a daily investment procedure through short-selling, at each trading day not all B-stocks in the big pool will have their causes occur. Accordingly, at each trading day, only those B-stocks in the big pool that have their respective causes occur will be included in the actual 
investment pool (small pool). Moreover, at each trading day, similar with [3]-[4], only those B-stocks in the small pool that are $P^{B}-T$ efficient which have their respective probabilities $\left(P^{B} \mathrm{~s}\right)$ above a threshold probability $(0.5+0.005 T)$ are added into the short-sell portfolio. The acceptable $P^{B}$ for each $T$ days, denoted by $P_{T}^{B}$, are $P_{T}^{B}=(0.505,0.51, \ldots, 0.6)$ for $T=1$ to 20 days. Accordingly, the proposed short-sell portfolio selection model is used to determine which stocks are to be included in the optimal short-sell portfolio.

\section{2.3. Short-Selling Portfolio Optimization Model}

From the generic scenario based safety-first (SF) portfolio selection model, considering $n$ stocks and $m$ scenarios, let the portfolio be denoted as $X=\left(x_{1}, x_{2}, \ldots, x_{n}\right) . x_{i}$ is the proportion of wealth invested on stock $i$ where $i=1,2, \ldots, n$. Scenario $j$ is represented by a row vector of return rates denoted as $\left(r_{1, j}, r_{2, j}, \ldots, r_{n, j}\right) . r_{i, j}$ is the return rate of stock $i$ when scenario $j(j=1,2, \ldots, m)$ occurs . If we consider $p_{j}$ as the likelihood of scenario $j$ to occur, the expected return rate of portfolio $X$ denoted as $E\left[R_{X}\right]$ is calculated as $E\left[R_{X}\right]=$ $\sum_{j=1}^{m} p_{j} R_{X_{j}} . R_{X_{j}}$, calculated as $R_{X_{j}}=\sum_{i=1}^{n} x_{i} r_{i, j}$, is the return rate of portfolio $X$ at scenario $j$. As for the SF portfolio selection model parameters, $R_{L}$ represents the threshold return rate, and $\gamma$ represents the threshold probability for $R_{L}$ to occur. Accordingly, the generic SF portfolio selection model is written as:

$$
\begin{array}{cl}
\operatorname{Max} & E\left[R_{X}\right]=\sum_{j=1}^{m} p_{j} R_{X_{j}} \\
\text { s.t. } & R_{X_{j}}=\sum_{i=1}^{n} x_{i} r_{i, j} \\
& R_{L}-R_{X_{j}} \leq M \omega_{j} \\
& \sum_{j=1}^{m} p_{j} \omega_{j} \leq \gamma \\
& 0 \leq x_{i} \leq 1 \\
& \omega_{j} \text { is binary } \\
i=1,2, \ldots, n ; j=1,2, \ldots, m
\end{array}
$$

The objective function (1) implies the maximization of the expected return. (2) is the return of the portfolio $X$ at scenario $j$ by the summation of the product of the portfolio weights multiplied to the return of the stocks at scenario $j . M$ is a large enough number that makes $\omega_{j}$ in (3) and (4) equal to 1 $\left(\omega_{j}=1\right)$ when $R_{X_{j}} \leq R_{L}$ and equal to $0\left(\omega_{j}=0\right)$ when $R_{X_{j}}>R_{L}$. (3) and (4) ensure that the risk $\left(\sum_{j=1}^{m} p_{j} \omega_{j}\right)$ of having an expected portfolio return rate $\left(E\left[R_{X}\right]\right)$ below the threshold return rate $\left(R_{L}\right)$ is under the threshold probability $(\gamma)$.

Since this study focuses on exploiting the short-sell B-stocks, we modify the generic SF model accordingly. In a short-selling investment, in order to have positive returns, we want to maximize the negative price change of all B-stocks within the portfolio. With respect to the objective function of the generic SF model, instead of maximizing the expected returns, we want to minimize it. Thus, (1) is now replaced with $\operatorname{Min} E\left[R_{X}\right]$. As for the safety-first or SF constraints (3) and (4), since it is desired that the B-stocks in the portfolio should have their stock prices drop, then instead of limiting the number of times when $R_{X_{j}}$ falls below $R_{L}$, it is now desired to have a high number of times when $R_{X_{j}}$ falls below $R_{L}$ as much as possible. Furthermore, it is also desired that the total probability of having a portfolio return falling below
$R_{L}$ is set to a minimum. Appropriately, (4) is now replaced with $\sum_{j=1}^{m} p_{j} \omega_{j} \geq \gamma$. Moreover, similar with [3]-[4], it is also desired to have a $P B-T$ efficient portfolio, such that only B-stocks with $P_{i}^{B}$ s satisfying $P_{i}^{B} \geq \alpha+\beta T_{i}(\alpha=0.5 ; \beta=$ $0.005)$ are included into the portfolio, such that $P_{T}^{B}=$ $(0.505,0.51, \ldots, 0.6)$ for $T=1$ to 20 days. Let $z_{i}$ be a binary indicator indicating that a B-stock satisfies the above condition where $z_{i}=1, P_{i}^{B} \geq \alpha+\beta T_{i}$ and $z_{i}=0$, otherwise. Accordingly, $z_{i}$ can be determined with the help of the two equations: $z_{i} \leq 1+\frac{1}{M}\left(P_{i}^{B}-\left(\alpha+\beta T_{i}\right)\right)$; $z_{i} \geq$ $\frac{1}{M}\left(P_{i}^{B}-\left(\alpha+\beta T_{i}\right)\right)$. Subsequently, the return of portfolio $X$ at scenario $j$ can now be calculated as $R_{X_{j}}=\sum_{i=1}^{n} z_{i} x_{i} r_{i, j}$. Subsequently, the proposed short-selling B-stock portfolio optimization model is written as:

$$
\begin{gathered}
\text { Min } E\left[R_{X}\right]=\sum_{j=1}^{m} p_{j} R_{X_{j}} \\
\text { s.t. } z_{i} \leq 1+\frac{1}{M}\left(P_{i}^{B}-\left(\alpha+\beta T_{i}\right)\right) \\
z_{i} \geq \frac{1}{M}\left(P_{i}^{B}-\left(\alpha+\beta T_{i}\right)\right) \\
R_{X_{j}}=\sum_{i=1}^{n} z_{i} x_{i} r_{i, j} \\
R_{L}-R_{X_{j}} \leq M \omega_{j} \\
\sum_{j=1}^{m} p_{j} \omega_{j} \geq \gamma \\
0 \leq x_{i} \leq 1 \\
z_{i} \text { and } \omega_{j} \text { are binary } \\
i=1,2, \ldots, n ; j=1,2, \ldots, m
\end{gathered}
$$

In (8), the return rate of the portfolio is minimized, which in turn maximizes the negative price change of the B-stocks in the portfolio, which is profitable to the short-selling investor. (9) and (10) ensure that $P i^{B} \geq \alpha+\beta T_{i}$ is satisfied such that only $P^{B}-T$ efficient short-sell B-stocks are included into the portfolio. With (9) and (10) working together, when $P_{i}^{B} \geq \alpha+\beta T_{i}$ (9) makes $z_{i}$ equal to 0 (unwanted 0 ) or 1 while (10) makes $z_{i}$ equal to 0 or 1 (unwanted 1), when $P_{i}^{B}<\alpha+\beta T_{i}$ (9) makes $z_{i}$ equal to 0 (desired 0) while (10) makes $z_{i}$ equal to 1 (desired 1 ), forcing $z_{i}$ to be $z_{i}=1$ only when $P_{i}^{B} \geq \alpha+\beta T_{i}$ and $z_{i}=0$ only when $P_{i}^{B}<\alpha+\beta T_{i}$. In (12), $M$ is a large enough number that makes $\omega_{j}$ in (12) and (13) equal to $1\left(\omega_{j}=1\right)$ when $R_{X_{j}} \leq R_{L}$ and equal to 0 ( $\left.\omega_{j}=0\right)$ when $R_{X_{j}}>R_{L}$. (12) and (13) ensure that the probability $\left(\sum_{j=1}^{m} p_{j} \omega_{j}\right)$ of having an expected portfolio return rate $\left(E\left[R_{X}\right]\right)$ below the threshold return rate $\left(R_{L}\right)$ is above the threshold probability $(\gamma)$. Accordingly in this study, following the works [1]-[4], the corresponding $P^{B}-T$ and SF parameters are set as $\left(P_{i}^{B} \geq 0.5+0.005 T_{i}\right)$ and $\left(R_{L}=-2 \%\right.$ and $\gamma=2 \%)$, respectively.

\section{EMPIRICAL RESULTS}

\section{A. Data Description}

The closing prices and volumes of 888 TWSE stocks were collected using the Taiwan Economic Journal (TEJ). Following the works of [1]-[4], the data obtained are then used to determine the behavioral stocks (B-stocks), but this time we focus on those B-stocks that will more likely have negative returns after the time-to-effect (T-days) or short-sell B-stocks. Accordingly, the identified short-sell B-stocks are 
included in the big pool, and at each trading day of the back-test, those short-sell B-stocks that have their respective causes occur are included for the investment pool (small pool) for that particular day. The data collected is from January 1, 1991, to June 30, 2017. We perform 30 months of back-tests with test period from December 2014 to May 2017. The investment strategy is to short-sell stocks within each month where rebalancing is only allowed from the first to the last trading day of the month. The return for the entire month is then calculated after all short sold stocks are already returned. The threshold return level $R_{L}$ is $R_{L}=-2 \%$ and the minimum probability for $R_{L}$ is set at $2 \%$. The $P^{B}-T$ parameters are set as $\alpha=0.5$ and $\beta=0.005$. Overall we have 2 portfolios that are compared to one another. We denote the resulting portfolio from the proposed optimization model as Short-Sell and denote the investment benchmark portfolio (market index) as Market. This study uses past 500 historical returns as the return scenarios and assumed equal likelihood for each scenario for the next 20 days.

\begin{tabular}{ccc} 
Distribution of Cumulative Returns & Short-Sell & Market \\
\hline Ending Cumulative Return & 2.1357 & 0.1080 \\
No. of Positive Cumulative Returns & 30 & 18 \\
No. of Negative Cumulative Returns & 0 & 12 \\
P-value (One-Proportion Test $>0.5)$ & $0.0000^{* * *}$ & 0.3620 \\
\hline
\end{tabular}

$* * *, * *$, and * respectively represent statistical significance at $0.01,0.05$, and 0.1 level

From Table 1, the empirical result shows that the short-selling portfolio can outperform the market portfolio over the 30 months of back-testing from December 2014 to May 2017. The Short-Sell portfolio has a significantly higher mean monthly return than the Market. Its corresponding standard deviation or volatility on the monthly returns is also higher than the market, meaning the Short-Sell portfolio is a riskier investment but with higher profitability reward (see Fig. 1). Comparing the pair-difference of the monthly returns after removing the outliers, although only close to being significant (P-value of 0.1260), we have a pair-difference value of 0.0445 . (The respective null hypothesis and the alternative hypothesis of the pair t-test of the pair-difference

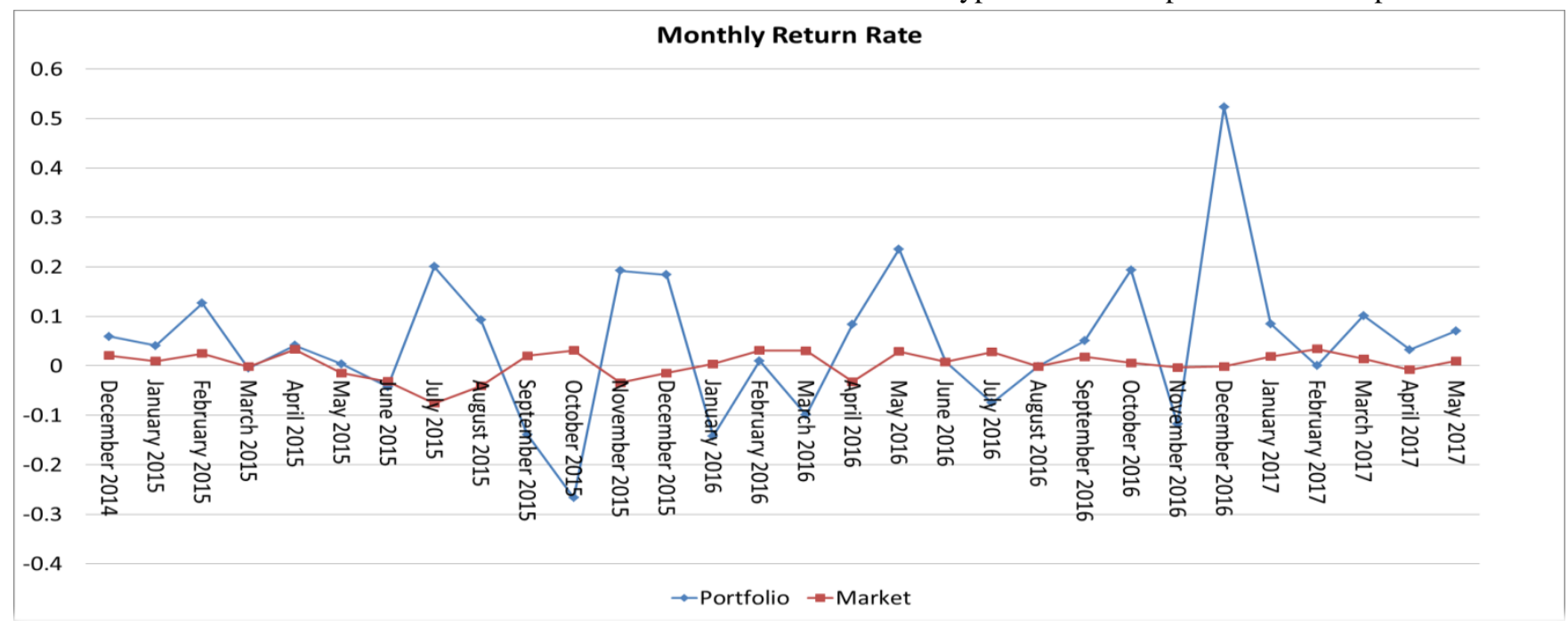

Fig. 1. Monthly return rates of the portfolios from December 2014 - May 2017.

\section{B. Back-Test Results}

In evaluating the performance of the short-selling portfolio, we compare the monthly returns of the portfolio against the market. It is assumed that with the help of short-sell B-stocks, the portfolio will be able to outperform the index performance of the market. This study compares the portfolios' monthly descriptive statistics, pair-difference on the monthly returns, distribution of the monthly returns, and their corresponding cumulative returns. The following comparisons are shown in Table 1 . The monthly return rates and cumulative returns of the portfolio are shown in Fig. 1 and Fig. 2, respectively.

TABle I. MONTHLy RETURn StATISTIC OVER 30 MONTHS OF BACK-TEST

\begin{tabular}{ccc}
\hline Monthly Return Statistics & Short-Sell & Market \\
\hline Mean Return & 0.0483 & 0.0038 \\
Standard Deviation & 0.1456 & 0.0263 \\
Pair-Difference of Returns & & 0.0445 \\
P-value (Pair-T test on Pair Difference) & & 0.1260 \\
\hline Distribution of Monthly Returns & Short-Sell & Market \\
\hline No. of Positive Returns & 21 & 18 \\
No. of Negative Returns & 9 & 12 \\
P-value (One-Proportion Test > 0.5) & $0.0430^{* *}$ & 0.3620 \\
\hline
\end{tabular}

of the returns are: $H_{0}$ : the pair difference of returns is less than or equal to $0 ; H_{A}$ : the pair difference of returns is higher than 0 ). This means that the short-selling portfolio in average has returns 0.0445 higher than the market. After analyzing the distribution of the monthly returns, both portfolios have more positive returns than negative returns, but the short-selling portfolios have more positive returns than the market portfolio. Furthermore, performing a one-proportion test (with an $H_{A}$ : the number of positive cumulative returns is over $50 \%$ of the total months of the test period) on the number of positive returns over the 30 months, the Short-Sell portfolio has a significant (P-value of 0.0430) number of positive returns (21) at 0.05 level while the market (18) is not significant (P-value of 0.3620). Subsequently, observing the cumulative returns over the 30 months, the Short-Sell portfolio has a $213.57 \%$ increase in the initial wealth while the Market portfolio only has a $10.80 \%$ increase. This further shows the capability of the Short-Sell portfolio to be more profitable than the Market portfolio. If we observe the monthly cumulative returns of both portfolios in Fig. 2, we can see that over the test period the Short-Sell portfolio have cumulative returns that are always above the market. 


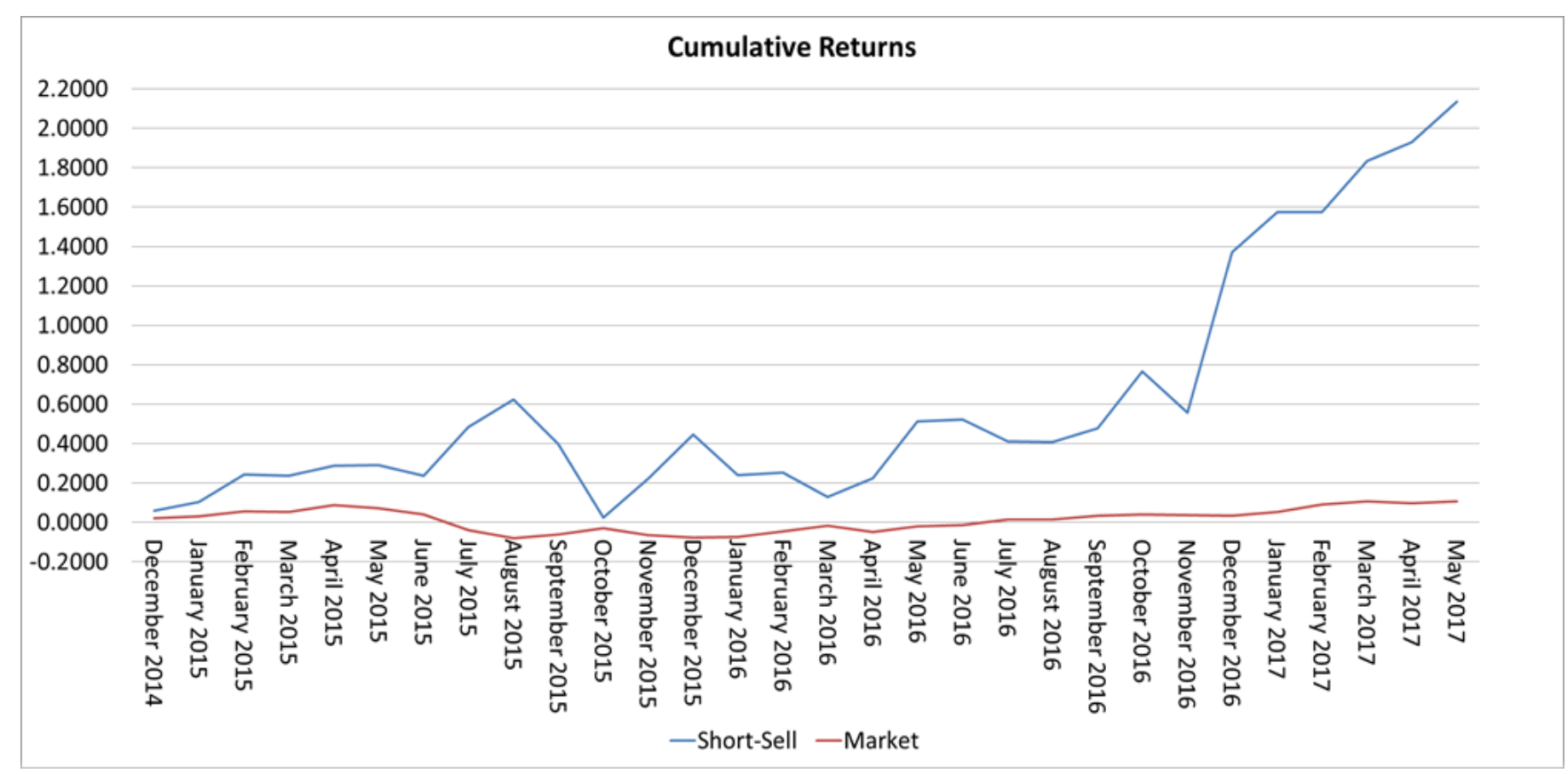

Fig. 2. Cumulative returns of the portfolios from December 2014 - May 2017

Moreover, it is evident from the distribution of these cumulative returns, that the Short-Sell portfolio outperforms the Market portfolio. The Short-Sell portfolio has positive cumulative returns throughout the 30-month period, while the Market has 12 days with negative cumulative returns. In addition, performing a one-proportion test on the number of positive cumulative returns for both portfolios, the Short-Sell portfolio is significant (P-value of 0.0000$)$ at 0.01 level with 30 days of positive cumulative returns, while the Market portfolio is not significant (P-value of 0.3620$)$ with only 18 days of positive cumulative returns. (The respective null hypothesis and the alternative hypothesis of the one-proportion tests on the number of positive cumulative returns are: $H_{0}$ : the percentage of the number of positive cumulative returns is less than or equal to $50 \% ; H_{A}$ : the percentage of the number of positive cumulative returns is greater than 50\%). Overall, from these results, we can conclude that the Short-Sell portfolio can really outperform the Market portfolio.

\section{Limitations of the Study}

It is to be noted that the study has several limitations that need to be addressed in future studies. First, in the empirical tests, only short-selling is considered. This means that the investment strategy is limited and contrary to the usual majority who buys-hold-sell assets. This is not necessarily a bad thing, but it does not reflect real investment conditions where investors can put long and short positions on assets. Intuitively, future tests should consider both long and short positions in order to reflect actual investments.

Second, since only 2 types of short-sell B-stocks are considered, the investment activity is only limited when there are causes of short-sell B-stocks spotted. If there are no short-selling causes for the over-reaction and disposition effect short-sell B-stocks (high positive return for over-reaction short-sell B-stocks; high positive cumulative return and high abnormal volume for disposition effect short-sell B-stocks) spotted, portfolio rebalancing is not performed. This means that the extra information of other possible types of short-sell B-stocks can't be exploited to maximize potential portfolio returns. This issue can be easily addressed by identifying operational definitions (ODs) of other irrational behaviors to identify other types of short-sell B-stocks. Appropriately, with the identification of other short-sell B-stocks, if long positions (buy, hold, and sell positions) are also considered then the corresponding non-short-sell B-stocks will also surely be of great help in generating superior portfolios.

Third, there are lots of theoretical investment strategies that show profitable results, but when costs are already considered these profits are eaten up by the transaction costs, taxes, commission costs and other fees. Similarly in this study, during the back-tests transaction costs were not considered. Therefore the proposed investment procedure may or may not work during real investment conditions with transaction costs and other charges. Future works should consider the transaction fees and other charges in order to really know if the current results will hold on actual investment conditions.

Lastly, other limitations to be addressed would be the limited back-test period (30 months) and the lack of statistical comparison to other traditional benchmark portfolios (mutual funds, exchange traded funds, etc.). By increasing the test-period of future tests (say 100 months or more), it can be known whether the profitability of the short-sell investment strategy will hold true for longer investment periods. Moreover, by comparing the portfolio to other traditional benchmarks, it can be known how the short-selling portfolio performs against other traditional investment benchmarks. Overall, addressing these issues on future studies will ensure that the resulting portfolio or investment strategy is surely worth the investment compared to other available investment options for investors in the market. 


\section{CONCLUSION}

The proposed variation of behavioral stock portfolio optimization model utilizes the cause-and-effect information on B-stocks, but instead of focusing on the irrationally affected stocks that will have the positive returns as the effects, this study exploits those B-stocks (short-sell B-stocks) that will have the effects of negative returns $(C A R \leq-1 \%)$ through short-selling. This study daily spot the causes of those short-sell B-stocks that will have the negative effects after their respective holding periods or time-to-effects ( $T$-days), from these stocks using the inverse of the generic safety-first portfolio selection model, the optimal portfolio is obtained. Each short-sold stock is returned only to the lender when the corresponding $T$-days is reached. The back-tests are done in a monthly basis, such that the short-selling of stocks are allowed on all trading days of each month and the corresponding monthly returns are calculated accordingly. From the empirical result of this preliminary study on short-selling B-stocks for 30 months from December 2014 to May 2017, it was observed that the result is consistent with the initial assumption that using short-sell B-stocks we can have a portfolio that is superior to the market portfolio. Moreover, with this promising result, this study concludes that short-selling B-stocks can also be considered as an alternative investment option for investors.

In summary, this preliminary study on short-selling B-stocks provides the following contributions: (1) exploited short-sell B-stocks which are expected to have the negative effect $(C A R \leq-1 \%)$ of irrational behavior(s) just like with the positive effect $(C A R \geq 1 \%)$ to have superior portfolios; (2) identified short-sell B-stocks of over-reaction and disposition effect; and (3) modified the generic safety-first portfolio selection model to cater portfolio optimization model through short-selling.

For further studies, the most appropriate extension is to have a portfolio selection model that considers both the usual buy-and-sell strategy and the short-selling strategy in exploiting B-stocks. This will make the portfolio selection model more realistic because at any time an investor will have both options of being able to buy or short-sell stocks (long and short positions). Furthermore, future studies should also consider the investment costs to have a more real-life result. Other extensions can consider other irrational behaviors in identifying more B-stocks. Also, they can further compare the portfolio performance with traditional investments like mutual funds, exchange-traded funds, and other investment benchmarks.

\section{ACKNOWLEDGMENT}

This work was partially supported by the Ministry of Science and Technology, Taiwan, ROC, under the grant contract MOST 105-2221-E-033-052.

\section{REFERENCES}

[1] K.-H. Chang, M. N. Young, M. I. Hildawa, I. J. R. Santos, and C. H. Pan, "Portfolio selection problem considering behavioral stocks," in Proc. the World Congress on Engineering vol. 2, 2015.

[2] K.-H. Chang and M. N. Young, "Behavioral portfolios of behavioral stocks with two-dimensional perception probability weights," Submitted for Review, 2016.

[3] K.-H. Chang, M.N. Young, and W. K. Lin, "Portfolio selection problem considering behavioral stocks under holding periods," International Journal of Modeling and Optimization, vol. 6, no. 4, 219, 2016.

[4] K.-H. Chang and M. N. Young, "Behavioral stock portfolio optimization considering holding periods of B-stocks," Submitted for Review, 2018.

[5] D. R. Cox and D. R. Peterson, "Stock returns following large one-day declines: evidence on short-term reversals and longer-term performance," The Journal of Finance, vol. 49, no. $1,255,1994$.

[6] J. Madura and N. Richie, "Overreaction of exchange-traded fuds during the bubble of 1998-2002," Handbook of Behavioral Finance: Edward Elgar Publishing Limited, 2010.

[7] H. Shefrin and M. Statman, "The disposition to sell winners too early and ride losers too long: theory and evidence," The Journal of Finance, vol. 40, no. 3, pp. 777-790, 1985.

[8] S. P. Ferris, R. A. Haugen, and A. K. Makhija, "Predicting contemporary volume with historic volume at differential price levels: Evidence supporting the disposition effect," The Journal of Finance, vol. 43, no. 3, pp. 677-697, 1988.

[9] A. Frazzini, "The disposition effect and underreaction to news," The Journal of Finance, vol. 61, no. 4, pp. 2017-2046, 2006.

[10] D. Kahneman and A. Tversky, "Subjective probability: A judgment of representativeness," Cognitive Psychology, vol. 3, no. 3, pp. 430-454, 1972.

[11] B. M. Barber and T. Odean, "Trading is hazardous to your wealth: The common stock investment performance of individual investors," The Journal of Finance, vol. 55, no. 2, pp. 773-806, 2000.

[12] B. M. Barber and T. Odean, "Boys will be boys: gender, overconfidence, and common stock investment," The Quarterly Journal of Economics, vol. 116, no. 1, pp. 261-292, 2001.

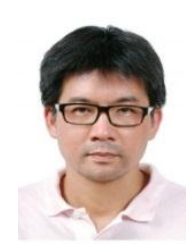

Kuo-Hwa Chang currently is a professor in the Department of Industrial and Systems Engineering in Chung Yuan Christian University, Taiwan. His current research interests include queueing system analysis on production systems and financial engineering.

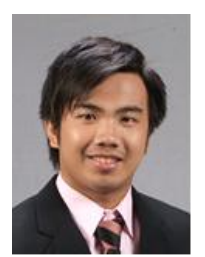

Michael N. Young is a Ph.D. candidate in the Department of Industrial and Systems Engineering in Chung Yuan Christian University, Taiwan. He is under the guidance of Dr. Chang and his research interests include financial engineering and queueing system analysis on production systems. He is currently listed as a faculty member of the School of Industrial Engineering and Engineering Management, Mapúa University.

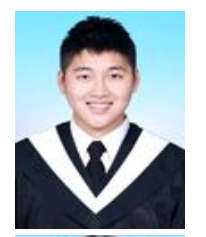

Chien-Chih Liu is a master student in the Department of Industrial and Systems Engineering in Chung Yuan Christian University, Taiwan. He is under the guidance of Dr. Chang and his research interest is financial engineering.

Hao-Ping Chung is a M.S. student in the Department of Industrial and Systems Engineering in Chung Yuan Christian University, Taiwan. She is under the guidance of Dr. Chang and her research interest is in financial engineering. 